\title{
Research on Aerospace Cooperative Continuation Observation Strategy for Maritime Moving Target
}

\author{
Liang Xingxing, Chen Chao, Li Jin, Zhang Mingxing, Cheng Gaungquan and Xiu Baoxin \\ Science and Technology on Information Systems Engineering Laboratory, National University of Defense Technology, Changsha 410073, P. \\ R. China.
}

\begin{abstract}
Maritime moving targets continuation observation has important implications to maintain the safety of the navigation barrier. Existing methods either rely on satellite observations, or only rely on UAV observations. Even though the two cooperative observations, it confined to a simple task allocation. They did not solve the problem of maritime moving targets continuation observation. On the basis of difference between satellites observation and UAV observation, we constructed the aerospace collaborative continuation observation model for maritime moving target, and put forward aerospace collaborative continuation observation strategy (ACCOS) to reduce the complexity of solving the model. According to the spatial and temporal characteristics of the task, ACCOS extracted five sub problems to solve the model: Satellite planning, UAV flight plan, UAV observation sequence, target potential area and distribution probability density prediction, UAV path planning. In order to realize the optimization goal of the model, the five sub problems are modelled and solved in turn. Finally, the simulation results show that the proposed method can greatly reduce the average observation period of the target, and effectively solve the problem of the continuous observation of maritime moving target.
\end{abstract}

\section{Introduction}

Due to the limitation of the horizon, it is difficult to continuously detect the long-range maritime moving target. Only using the upper unmanned aerial vehicle (UAV) observation, it will lead to blindly trap into the search for the lack of a priori information; only rely on satellites, it will get a cover on the target about less $20 \%$ observation time. Also, optical satellite usually is been affected by meteorological conditions, light illumination condition; and electronic satellite affected by the outside world is weak, but the positioning accuracy is poor, need to cooperate with other observation equipment.

There are many researches about the satellite observations of the moving target, for example, to study the various algorithms for moving target search by satellite [1], satellite observation area construction [2], the key technology of the multi satellite cooperation of observation planning [3] and so on. Relevant research to solve the problem of more fully, but the study itself is only limited to similar satellite search or to a stationary target observation, to only complete the target observation. They could not complete the continuous near real time observation of maritime moving targets.

Compared with fixed orbit satellite observation, UAV's observation effect is more flexible and better. It can be regarded as an important supplementary resources of satellite. At present, many research focus on the UAV real-time online planning. UAV research focuses on multi
UAV cooperative search method description [4], multi UAV search path optimization [5-6], the exchange of information between planning and search of multi UAV [7-9] and so on. These research made the assumption for the distribution of the targets that designated search area at first, and then find regional goals. This model is relatively simple, and could not effectively reflect and solve the real problem. For the sea area which is far from the mainland, existing detection method mostly use other means to obtain the target location, and then sent UAV to closely observe, the specific process is to estimate the potential area after finding the target, then to closely detect the targets. The existing methods are only qualitative analysis, and there is no appropriate model to quantify the analysis.

In conclusion, UAV and satellite observations have advantages and disadvantages, but the two can complement each other. Scholars put forward the multiplatform cooperative evolutionary algorithm, which is used to divide the observation mission [10]; to set up a space cooperation service planning model, the analysis of the aerospace collaborative work principle [11-12]; the key technologies of collaborative of the space for the detailed description [13-14]. But such research also only stay in the assignment of scheduling, they complete the regional division of the platform through the corresponding means, and on this basis, finish task planning of every platform. But their research target is static, and has not formed the true meaning of the joint. 
For continuous observation of maritime moving target, this paper constructs the collaborative space continuation observation model, puts forward the aerospace collaborative continuation observation strategy (ACCOS) to solve the model. On the basis of temporal characteristics of tasks, there is five sub problem solving models from ACCOS: Satellite planning, UAV flight plan, UAV sequence, target potential area and distribution probability density prediction, UAV path planning. After solving this five problems, getting the observation window of the satellite observation sequence set, UAV observation sequence, path planning results and trigger event time series. From the result, it reduced the average observation period of observation platform, solving the problem of continuous observation of maritime moving target.

The structure of the paper is as follows. In section II of the paper, maritime moving targets continuation observation model is provided, along with input, output, constraints and optimization objective. Section III outlines the aerospace collaborative continuation observation strategy. Several case studies are illustrated in Section IV, along with their results. Conclusions and future work are then provided in section $\mathrm{V}$.

\section{Problem description and model}

\subsection{Problem description}

The problem is described as continuous observation of maritime moving target. The target moves from single platform observation area to multi-platform observation area. The satellite observation information couldn't meet the needs of the target observations, need to send UAV to the target area, together with the satellite to complete continuous observation of moving targets. Therefore, the problem can be described for the moving target set Tar and satellites, according to the actual intention, send UAV to the target's potential regions to closely observe. In the process of observation, satellite sent observed target information to guide UAV, UAV found the optimal observation path according to the optimization objective, to complete the task of continuous observation for the targets.

\subsection{Model modelling}

Maritime moving targets continuation observation is a complex system engineering. The process involves multiple entity unit, output and constraints. So in this paper, an aerospace collaborative continuation observation model is made to describe the complex problem. Some definition is shown as follow.

\subsubsection{Input}

Tar $=\left\{\operatorname{tar}_{1}, \cdots, \operatorname{tar}_{N}\right\}:$ Observation target sets;

$\left(\right.$ Lon $\left._{\text {Base }}, L_{\text {Base }}\right)$ : Coordinate of UAV base; $\left(P_{i}, V_{i}\right)$ : Position and speed vector of target $\operatorname{tar}_{i}$;
Plat(Sat,UAV) : Observation platform, and Sat $=\left\{s a t_{1}, \cdots s a t_{N_{\text {sat }}}\right\} \quad$ is satellite resource set, $U A V=\left\{u a v_{1}, \cdots u a v_{N_{u a v}}\right\}$ is UAV resource set;

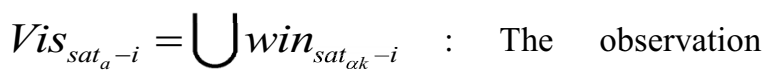
windows set of satellite $s a t_{\alpha}$ to target $\operatorname{tar}_{i}$, ordered by time, $w_{\text {sat }_{\alpha k}-i}=(w s, w e)$ is the start and end time of once observation.

\subsubsection{Output}

The target's observation windows and location precision set from all satellites:

$$
\text { SatVis }_{i}=\left\{\left(\text { win }_{i k}, \text { pre }_{i k}\right) \mid k=1,2, \cdots\right\}
$$

The observation sequence of $u a v_{i}$ :

$$
\operatorname{Seq}_{i}=\left\{\operatorname{tar}_{i 1}, \cdots \operatorname{tar}_{i k}, \cdots \operatorname{tar}_{i 1}\right\}
$$

The observation path of target $\operatorname{tar}_{i}$ :

$$
R_{i}=\left(P_{1}, P_{2}, \cdots, P_{n}\right)
$$

The trigger event time series:

$$
\text { EVENT }=\left\{\left(\text { time }_{i}, \text { event }_{i}\right) \mid i \in 1,2, \cdots\right\}
$$

time $_{i}$ is occurrence time of event event . $_{\text {. }}$

\subsubsection{Constraints and optimization objective}

Optimization objective for the continuous observation of maritime moving targets:

$$
\min \bar{T}=\frac{\sum_{i=1}^{N} T_{i}}{N}
$$

Constraints is:

$$
\begin{aligned}
& P_{i} \notin \text { Area } \\
& \alpha_{s a t_{i}}<\max \alpha_{s a t_{i}} \\
& \sum_{k=1}^{N_{\text {VAV }}} X_{i}^{k}=1, x_{i}^{\alpha}=\{0,1\} \\
& \sum_{k \in S e q_{i}} L\left(P_{k}, P_{k+1}\right)<f l y D \\
& \frac{\sum_{k \in \text { Seq }_{i}} L\left(P_{k}, P_{k+1}\right)}{\text { fly } V}<\text { flytime }
\end{aligned}
$$

$\alpha_{s a t}$ is the sensor's angle of optical satellite; $\max \alpha_{\text {sat }_{i}}$ is maximum sensor's angle of optical satellite; $L\left(P_{k}, P_{k+1}\right)$ is the distance between position $P_{k}$ and 
$P_{k+1} ; f l y D$ is maximum cruising distance of UAV; fly $V$ is maximum cruising speed of UAV; flytime is maximum cruising time of UAV.

Optimization objectives ensure target the average observation period of target is the shortest; Constraints (6) expressed in the UAV into the search, the target cannot enter one's own space; (7) said the satellite's swing angle can not exceed the maximum swing angle; and (8) said each target needs to be observed and belong to a UAV to observe; (9) said that the UAV cannot exceed the farthest cruising range; (10) said UAV cannot exceed the maximum flight time.

\section{Operational research}

Aerospace collaborative continuation observation model regards maritime moving target continuation observation problem as mathematical model, but the model itself is complex to solve. In order to overcome this problem, this paper proposes aerospace collaborative continuation observation strategy (ACCOS) to reduce the complexity of the model's solution. This section divided into two parts to study ACCOS: first is the overall aerospace collaborative design framework; the other is the specific description of ACCOS.

\subsection{Overall design}

Under aerospace collaborative maritime moving targets continuation observation involves multiple element, including the satellite, UAV, control center and so on. A complete task decision process usually is made by observe, orient, decide and act, namely the OODA. As time passing by, it constitutes a dynamic cycle. On a maritime moving target continuation observation also follow the OODA process. The design of the overall framework as shown in Fig. 1.

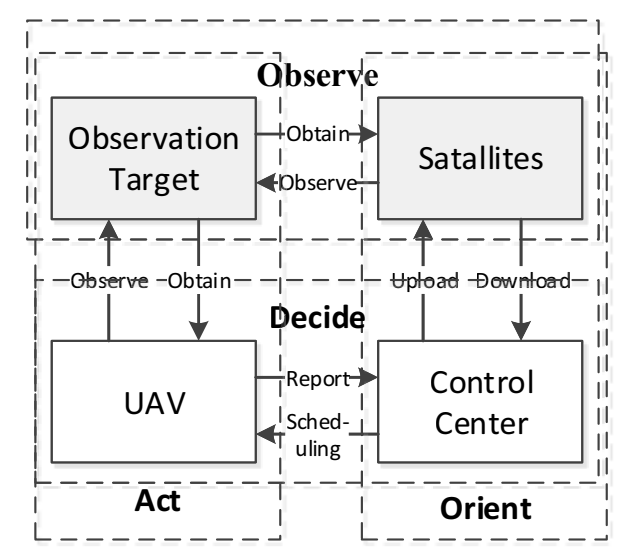

Figure 1. Overall design framework.

Fig. 1 describes the overall framework of maritime moving target continuation observation tasks by aerospace collaborative: 1 . electronic observation satellite and optical imaging satellite observe the observation area, transferring the regional information timely to the control center; 2. After satellite's observation information is sent to control center, it identifies suspicious target within the region, analyzes the target intention, judges whether adjust the satellite attitude. According to the relevant constraints, it is necessary to decide whether the UAV should fly closely observation area; 3 . Control center send UAV to observation area, setting the UAV track, and planning online; 4. According to the instruction information, UAV fly to the designated location. The cycle is a dynamic closed-loop process, effectively solving maritime moving targets continuation observation problem.

\subsection{Detailed design}

The aerospace collaborative overall design framework is given for maritime moving target continuation observation in last section, describing the information interaction relation between the various units, but have not yet specifically described strategies for each unit, and couldn't give the clear method to solve the model. Therefore, there is detailed analysis of the design for the ACCOS in this section.

\subsubsection{Strategy description}

ACCOS describes the strategy of various elements on the task timeline, that is, what the problem is, what strategy is been used.

The specific description of ACCOS is as follows.

(1) First, electronic satellite is used to detect the target. If the target is in the observation window, it detects the target and returns the result; otherwise, it continues to wait, and re-start the task.

(2) Secondly, satellite plans is made, according to the type of the next satellite. If it is optical satellite, we will analyse the obtained information, getting next arrival time interval and target potential area, calculating the swing angle of the optical imaging satellite attitude; otherwise, it continues to wait, and re-start the task.

(3) Next, analyzing the situation is essential while making satellite plans. If the analysis result shows that it is necessary to send UAV, the UAV flight plan should be made, including the number of sending UAV and UAV base; otherwise, it continues to wait, and re-start the task.

(4) When we decided to send the UAV, the UAV flight path should be modified according to satellite observations result. If UAV will arrive the target area, it is necessary to analyze the UAV observation sequence analysis; on the contrary, it is well to keep the cruise state and modify the UAV flight path.

(5) After finishing analyzing the UAV observation sequence, we need to determine whether the UAV is near the target. If it is, it is time to predict the distribution probability density of the target area; if not, it is ok to maintain the cruise state, and revise the route.

(6) Finally, when the forecast is over, UAV turns to the search for the target. When there is target which is still not observed in the observation sequence, UAV will turn to the next target observation; when all targets have been observed, UAV turns into the tracking observation, and repeats prediction - search mode. 


\subsubsection{Strategy implementation process description}

ACCOS describes a complete mission design: from the beginning of the satellite observation, to UAV turning into the target tracking observation.

ACCOS contains three planning: satellite-control centre planning (SCP), control centre -UAV planning (CUP), UAV autonomous planning (UAP). SCP contains the interaction between satellites and control centre; CUP contains the interaction between control centre and UAV; UAP shows UAV autonomous planning.

Along with the time axis, ACCOS decomposes problems and reduces the complexity of the model solution. According to the design of the ACCOS, there is five sub problems in the three kinds of planning: satellite planning, UAV flight plan, UAV observation sequence, potential target regions and the distribution probability density , UAV path planning.

(1) Satellite planning. Satellite planning is the beginning of the task, containing many kinds of satellites and control centre, and each satellite observation window is the starting point of the decision. The process specifically described as follows: electronic satellite finds the target in a large range observation area at first; when finding the target, control centre value the target's observation value, to determine whether optical imaging satellite plan to observe this goal, getting satellite observation result. The planning result of this stage is to get the observation window of the satellite to the target.

(2) UAV flight plan. UAV flight plan is the beginning of the UAV planning, containing control centre and UAV. The distance between the target and the base is far away. When UAV flew near the target, the target distribution has been changed. So the control centre needs to decide how much UAV should been send and gives the UAV cruise path. The results of this stage is the number of $\mathrm{UAV}$ and the cruise routes planning.

(3) UAV observation sequence. UAV observation sequence is the beginning of UAV searching. In UAV cruise, control centre constantly revises UAV's flight path according to the received information. When the UAV begin to carry out their tasks, control centre will start planning UAV observation sequence containing target assignment and observation sequence planning: the former divides the target into group according to the number of UAV, and the latter plans to get the observation sequence of each UAV for the assigned target. The result of this stage is the result of the target assignment and observation sequence of UAV.

(4) Target potential area and distribution probability density prediction. Getting potential target regions and the distribution probability density is the beginning of UAV autonomous planning. With the cooperation of the satellite, UVA make a reasonable prediction of the target distribution using the received information. The result of this stage is to get potential target regions and the distribution probability density.

(5) UAV path planning. According to the target distribution information predicted by the previous stage, UAV obtains the observation path of the target combining with the optimization goal. The result of this stage is to get UAV path planning for each UAV.

\section{Simulation example and result analysis}

At present, less research is in aerospace collaborative continuation observation, while more research is in the collaborative task scheduling. So there is no recognized benchmark test problem set to compare the algorithm running results. In order to verify the applicable and feasible of the method, we uses the STK to set a scene, four sets same orbit altitude electronic satellite and four groups same orbital altitude optical imaging satellite, while the amount of UAV is sufficient.

Algorithm used Visual Studio $\mathrm{C}++$ to achieve and run in microcomputer which is Intel Core $3.40 \mathrm{GHz}, 4 \mathrm{G}$ of memory; Using DBSCAN clustering algorithm with radius parameter $\varepsilon=3 \mathrm{~km}$ and neighborhood density threshold MinPts $=1$; target assignment is based on UAV number in the task by K-means clustering; single UAV observation sequence and actual flight path calculation used genetic algorithm with the crossover probability that is 0.8 and the mutation probability that is 0.2 calculated by several times; when the maximum evolution algebra or converge to the global optimum is reached, the algorithm stops.

In simulation process of this experiment, control center decided to send three UAVs based on the observation information, cooperating with the satellite to finish target continuation observation. When the satellite observes the target, it update the information to the UAV. Specific simulation response event schedule is shown in Table 1:

Table 1. The simulation events.

\begin{tabular}{|c|c|c|}
\hline Event & Time(s) & Description \\
\hline Target run into area & 2200 & Based on Constraints \\
\hline Send UAV & 2200 & Based on DBSCAN \\
\hline Satellite's Observation & 6254 & Optical satellite \\
\hline Target assignability & 6637 & Based on K-means \\
\hline Satellite's Observation & 8928 & Electronic satellite \\
\hline UAV1 turns into tracing & 9462 & None \\
\hline UAV2 turns into tracing & 9500 & None \\
\hline UAV0 turns into tracing & 9700 & None \\
\hline
\end{tabular}

Table 2. The satellite observation ability.

\begin{tabular}{|c|c|c|c|}
\hline Number & $\begin{array}{c}\text { Observation } \\
\text { Window }\end{array}$ & $\begin{array}{c}\text { Duration } \\
\text { Time }\end{array}$ & $\begin{array}{c}\text { Positioning } \\
\text { Accuracy }\end{array}$ \\
\hline 1 & $2181.9-3142.6$ & 960.753 & 0 \\
\hline 3 & $3363.3-3764.3$ & 400.996 & 0 \\
\hline 6 & $6254.2-6637.9$ & 383.707 & 1 \\
\hline 1,8 & $8928.3-9624.0$ & 695.679 & 0.07 \\
\hline 5,3 & $9632.6-10051.2$ & 418.575 & 0.41 \\
\hline 7 & $\begin{array}{c}12252.9- \\
12634.7\end{array}$ & 381.886 & 1 \\
\hline 3 & $\begin{array}{c}16511.9- \\
17188.4\end{array}$ & 676.512 & 0 \\
\hline 2 & $\begin{array}{c}19822.0- \\
20615.2\end{array}$ & 793.277 & 0 \\
\hline 2 & $\begin{array}{c}26412.2- \\
27359.6\end{array}$ & 947.407 & 0 \\
\hline \multicolumn{2}{|c|}{ Average Duration Time } & \multicolumn{2}{|c|}{$639.57 \mathrm{~s}$} \\
\hline \multicolumn{2}{|c|}{ Average Interval Time } & \multicolumn{2}{|c|}{0.33} \\
\hline \multicolumn{2}{|c|}{ Average Positioning } \\
Accuracy
\end{tabular}


In this paper, we use multiple types of satellite observations for the target, and the duration is about 26400s (7h), from the beginning of satellite observation to track the target by UAV. The observation results are shown in Table 2. Number 1-4 represents satellite electronic observation satellite, and 5-8 means optical imaging satellite; Observation Window is said that target could be observed by satellites in this time; Duration Time represents the size of the observation window; Positioning Accuracy means satellite's capacity where optical is 1 and electronic is 0 .

Through the analysis of the results in Table 2, we could see the lack prediction of satellite observations. By only relying on satellites, coverage of satellite observation is only $20.8 \%$. In this experiment, Target's speed is $50 \mathrm{~km} / \mathrm{h}$. In the blank space of observation, target's movement range is $1122.2 \mathrm{~km} 2$, and moving target range is very wide. Therefore, it is essential to use UAV cooperating with satellite for achieving target continuation observation.

After UAV taking-off, according to the target distribution and real distance, control center determine whether to start planning for UAV observation sequence. The simulation of the observation sequence for UAV is shown in Table 3: Number means the UAV number; Observation Sequence represents UAV observations sequence, and the serial number of the first goal means the UAV search first goal; Flying Time represents the time interval for the target observation.

Table 3. UAV observation sequence.

\begin{tabular}{|c|c|c|c|}
\hline Number & $\begin{array}{c}\text { Observation } \\
\text { Sequence }\end{array}$ & $\begin{array}{c}\text { Optimization } \\
\text { Objective }\end{array}$ & $\begin{array}{c}\text { Flying } \\
\text { Time }\end{array}$ \\
\hline 0 & $9-10-9$ & $240.13 \mathrm{~km}$ & $86.4 \mathrm{~s}$ \\
\hline 1 & $1-5-6-2-0-1$ & $248.43 \mathrm{~km}$ & $172.8 \mathrm{~s}$ \\
\hline 2 & $8-3-4-7-8$ & $245.37 \mathrm{~km}$ & $130.46 \mathrm{~s}$ \\
\hline
\end{tabular}

As we can see from the above analysis, due to the different target distribution and UAV observation sequence, observation gaps isn't same and maximum flying time is $130.46 \mathrm{~s}$. Compared to satellite observations, UAV observation's accuracy and level of detail has also been improved.

Based on the above analysis, in space cooperation, the ability of two types of resources has been fully applied, improving the average observation period from $2425 \mathrm{~s}$ to $141.7 \mathrm{~s}$, solving the problem that target run into area by only UAV observation.

\section{Conclusion}

Because of the different focus of observation type, continuous observation of maritime moving target hasn't been efficiently solved. This paper constructs the maritime moving target continuation observation model, puts forward the aerospace collaborative continuation observation strategy (ACCOS) to solve the model. On the basis of temporal characteristics of tasks, getting sub problem solving models from ACCOS: Satellite planning, UAV flight plan, UAV sequence, target potential area and distribution probability density prediction, UAV path planning. After solving this five problems, we get the observation window of the satellite observation sequence set, UAV observation sequence, path planning results and trigger event time series. From the result, we found that it improved the average observation period.

\section{Acknowledgment}

This project was supported partially by National Natural Science Foundation of China (71471174).

\section{References}

1. Y. Ci, Y. Xu, Y. Tan. Comparison of Several Algorithms for Maritime Moving Target Search by Satellite[J]. Acta Armamentarii, 1: 029, (2009).

2. C. Ran, H. Wang, G. Xiong, et al. Research on Mission-Planning of Ocean Moving Targets Imaging Reconnaissance Based on Improved Genetic Algorithm [J]. Journal of Astronautics, 2: 025, (2010).

3. Y. Guo. Research on key techonology research for mulit-type satellitestask planning [D]. National University of Defense Technology, (2009).

4. Y. Altshuler, V. Yanovsky, I. A. Wagner, et al. Efficient cooperative search of smart targets using uav swarms[J]. Robotica, 26(04): 551-557, (2008).

5. P. B. Sujit, D. Ghose. Search using multiple UAVs with flight time constraints[J]. Aerospace and Electronic Systems, IEEE Transactions on, 40(2): 491-509, (2004).

6. Y. Zhang. The UAV formations Research based on probability calculations and state predicted [D]. Dalian University of Technology, (2013).

7. Y. Xuan, C. Huang, W. Wu, et al. Coverage search strategies for moving targets using multiple unmanned aerial vehicle teams[J]. Systems Engineering and Electronics, 35(3): 539-544, (2013).

8. J. Tisdale, Z. Kim, J. K. Hedrick. An autonomous system for cooperative search and localization using unmanned vehicles[C]//AIAA Guidance, Navigation and Control Conference and Exhibit, Honolulu, HI: 18-21, Aug. (2008).

9. J. George, P. B. Sujit, J. B. Sousa. Search strategies for multiple UAV search and destroy missions[J]. Journal of Intelligent \& Robotic Systems, 61(1-4): 355-367, (2011).

10. G. Bai, L. Xing, R. He, st al. Scheduling multiplatforms collaborative observation based on cooperative evolution $[\mathrm{J}]$. Journal of National University of Defense Technology, 35(4): 182-188, (2013).

11. J. Li, Z. Zhong, W. Hu, et al. A service model based on SWE for space-aeronautics cooperation earth observing operations[J]. Journal of National University of Defense Technology, 35(3): 108-113, (2013)

12. J. Li, Z. Zhong, N. Jing, et al. Space-air Resources Multi-phase Cooperation Task Planning Approach Based on Heterogeneous MAS Model[J]. Acta 
Aeronautica ET Astronautica Sinica, 34(7): 16821697, (2013).

13. J. Yu. Research on Key Technologies of Cooperative Task Scheduling for Airborne and Spaceborne Earth
Observing Assets[D]. National University of Defense Technology, (2011).

14. J. Bai, Y. Wen. Multi-sensor Cooperative Detection Technology for Sea Surface Targets[J]. Radio Engineering, 45(3): 10-13, (2015). 